\title{
Measurement of WW and WZ production with one vector boson decaying hadronically at ATLAS
}

\author{
Brian Edward LINDQUIST ${ }^{* \dagger}$ \\ ( Stony Brook University (US)) \\ E-mail: brian.edward.lindquistecern.ch
}

\begin{abstract}
A measurement by ATLAS of the combined WW and WZ cross-section in proton-proton collisions at the LHC at $\sqrt{s}=7 \mathrm{TeV}$ is presented, using $4.7 \mathrm{fb}^{-1}$ of data. The measurement is performed in the $\ell v j j$ final state by fitting the di-jet invariant mass distribution. Evidence for $\mathrm{WW} / \mathrm{WZ}$ production is found at the $3.3 \sigma$ level, and a cross-section of $\sigma(p p \rightarrow W W / W Z)=72$ \pm 9 (stat.) \pm 15 (syst.) \pm 13 (Monte Carlo stat.) pb is measured, consistent with the Standard Model expectation.
\end{abstract}

XXI International Workshop on Deep-Inelastic Scattering and Related Subjects 22-26 April, 2013

Marseilles, France

${ }^{*}$ Speaker.

${ }^{\dagger}$ On behalf of the ATLAS Collaboration. 


\section{Introduction}

Diboson production ( $p p \rightarrow V V^{\prime} ; V, V^{\prime}=W$ or $Z$ ) is an important process at the Large Hadron Collider (LHC). Measurements of the total cross-section and kinematic distributions of diboson production provide a powerful test of the electroweak (EW) part of the Standard Model (SM). Diboson production can proceed through either t-channel or s-channel diagrams. The latter are due to triple gauge couplings that are precisely predicted in the SM; diboson measurements are thus sensitive to anomalous triple gauge couplings that can occur in the context of many different models of new physics. Another reason to study diboson processes is that they are important backgrounds to many physics searches, such as the $H \rightarrow W^{+} W^{-}$search. Lastly, $V V^{\prime}$ measurements can be viewed as "stepping-stone" measurements towards ultimate measurements of vector-boson scattering ( $p p \rightarrow V V^{\prime} j j$, where $j$ is a jet), which are crucial to a better understanding of EW symmetry breaking.

The processes $p p \rightarrow W^{+} W^{-}, W Z$, and $Z Z$ have all been measured previously at the LHC, by both ATLAS and CMS [1-7]. However, most of these processes have only been measured in the fully leptonic $\left(W \rightarrow \ell v, Z \rightarrow \ell^{+} \ell^{-}\right.$) channels. An intriguing complementary measurement is the semi-leptonic channel $p p \rightarrow W W \rightarrow \ell v j j$, which has a rate about six times larger than $W W \rightarrow \ell v \ell v$. Additionally, it allows for better reconstruction of the event kinematics than in $W W \rightarrow \ell v \ell v$ decays, due to the presence of only one neutrino rather than two. However, since the experimental di-jet mass resolutions at ATLAS and CMS are insufficient to distinguish $W \rightarrow j j$ from $Z \rightarrow j j$ decays, it is necessary to measure the combined process $p p \rightarrow(W W / W Z) \rightarrow \ell v j j$. A further complication in this channel is the large background, coming primarily from $W+$ jets events.

In these proceedings, we report a measurement by ATLAS of the $p p \rightarrow(W W / W Z) \rightarrow \ell v j j$ cross-section at $\sqrt{s}=7 \mathrm{TeV}$, using $4.7 \mathrm{fb}^{-1}$ of proton-proton collision data [8]. The measurement is performed by fitting the di-jet mass spectrum of events containing an $\ell(=\mu, e)$ and large missing transverse energy, and looking for a peak from $W / Z \rightarrow j j$ decays. A similar measurement was recently reported by the CMS Collaboration [9].

\section{Selection and Background Modeling}

The main backgrounds in this analysis come from $V+$ jets $(V=W, Z), t \bar{t}$, single-top, and multijet processes. The $V+$ jets background is modeled with Alpgen interfaced to Herwig and Jimmy. The $t \bar{t}$ and single-top backgrounds are modeled with MC@NLO. The multi-jet background is modeled using a data-driven technique described later in this section. The signal $W W$ and $W Z$ processes are modeled with Herwig, and normalized to the NLO cross-sections of $44.9 \pm 2.2 \mathrm{pb}(W W)$ and $18.5 \pm 1.3 \mathrm{pb}$ (WZ) obtained with MCFM [10]. The Monte Carlo (MC) simulated events are passed through a full GEANT4 [11] simulation of the ATLAS detector. Effects due to multiple interactions per bunch crossing are included in the simulation. A detailed description of the detector can be found in Ref. [12].

Events in this analysis must contain one high- $p_{\mathrm{T}}$ electron or muon candidate, large missing transverse energy $\left(E_{\mathrm{T}}^{\mathrm{miss}}\right)$, and exactly two jets, and are also required to pass a high- $p_{\mathrm{T}}$ muon (18 $\mathrm{GeV})$ or electron $(20-22 \mathrm{GeV})$ trigger. Electron and muon candidates are required to have a $p_{\mathrm{T}}$ 
of at least $25 \mathrm{GeV}$ and electrons must have a pseudorapidity $|\eta|$ less than 2.47 and excluding the calorimeter crack region $1.37<|\eta|<1.52$, whereas muons must have $|\eta|<2.4$. Lepton candidates are required to satisfy both track and calorimeter isolation criteria, and are required to have tracks pointing back to the primary vertex. Jets are formed with the anti- $k_{T}$ algorithm [13] with a radius of 0.4 , using calorimeter clusters calibrated at the electromagnetic scale. Jets are required to have a jet vertex fraction greater than 0.75 , defined as the scalar sum of the $p_{\mathrm{T}}$ of the tracks associated to the jet that point to the primary vertex, divided by the scalar sum of the $p_{\mathrm{T}}$ of all the tracks associated to the jet.

Events are required to have one electron or muon candidate with $p_{\mathrm{T}}>25 \mathrm{GeV}$, and the event is vetoed if there is an additional lepton with $p_{\mathrm{T}}>20 \mathrm{GeV}$. An event must have one jet with $p_{\mathrm{T}}>30 \mathrm{GeV}$ and a second jet with $p_{\mathrm{T}}>25 \mathrm{GeV}$, and in order to reduce $t \bar{t}$ backgrounds, events are rejected if there is an additional jet with $p_{\mathrm{T}}>20 \mathrm{GeV}$. Each event must have a $E_{\mathrm{T}}^{\text {miss }}>30$ $\mathrm{GeV}$ and the transverse mass $m_{T}$, formed from the $E_{\mathrm{T}}^{\text {miss }}$ and transverse momentum of the lepton, must satisfy $m_{T}>40 \mathrm{GeV}$. The difference in azimuthal angle $\Delta \phi$ between the $E_{\mathrm{T}}^{\text {miss }}$ and the highest$p_{\mathrm{T}}$ jet must be greater than 0.8 , to reduce multi-jet backgrounds, and each jet must be separated from the lepton by $\Delta R(j, \ell)>0.5$, where $\Delta R \equiv \sqrt{\Delta \phi^{2}+\Delta \eta^{2}}$. The following additional angular requirements between the leading and sub-leading jets $\left(j_{1}\right.$ and $\left.j_{2}\right)$ are imposed: $\Delta \eta\left(j_{1}, j_{2}\right)<1.5$ and $\Delta R\left(j_{1}, j_{2}\right)>0.7$.

The multi-jet background is estimated from data. These backgrounds can be due to jets being misreconstructed as electrons, or due to semileptonic decays from heavy-flavor jets. Control regions are created that are enriched in multi-jet backgrounds, by applying the normal selection except inverting lepton quality criteria. For the electron channel, the electron candidates are required to have no signal in the Transition Radition Tracker [12], and for the muon channel, the fakemuon candidates must fail to point to the primary vertex. These control regions are used to form $E_{\mathrm{T}}^{\text {miss }}$ templates for the multi-jet background. Then in the nominal selection region, the $E_{\mathrm{T}}^{\text {miss }}$ cut is removed, and a fit is performed to the $E_{\mathrm{T}}^{\text {miss }}$ spectrum using the $E_{\mathrm{T}}^{\text {miss }}$ templates derived previously, and the multi-jet normalization is extracted. The V+jets background is also allowed to float in this fit, from which a scale-factor is derived that is used for an initial data-MC comparison, shown in Fig. 1. As can be seen, the data is well described by MC expectation.

\section{Fit Method and Results}

The $W W / W Z$ cross-section is measured using a binned maximum-likelihood fit to the dijetmass distribution, from [25-250] GeV in 5-GeV bins. The electron and muon channels are fit separately in a simultaneous fit. Dijet-mass templates are created for the signal and for three background categories: V+jets, $t \bar{t} /$ single-top, and multi-jet, and are shown in Fig. 2. To take into account the uncertainty on the background rates, the $\mathrm{V}+\mathrm{jets}$ and top backgrounds are allowed to float in the fit, subject to $20 \%$ and $10 \%$ constraints, respectively. The multi-jet background normalization is fixed in the nominal fit, and varied as a systematic. Nuisance parameters are introduced that account for the effects of certain systematics, most importantly the jet energy resolution and the shape of the V+jets background (estimated by varying parameters in the Alpgen generation), and these nuisance parameters are allowed to float in the fit. Other systematics are estimated using pseudo- 

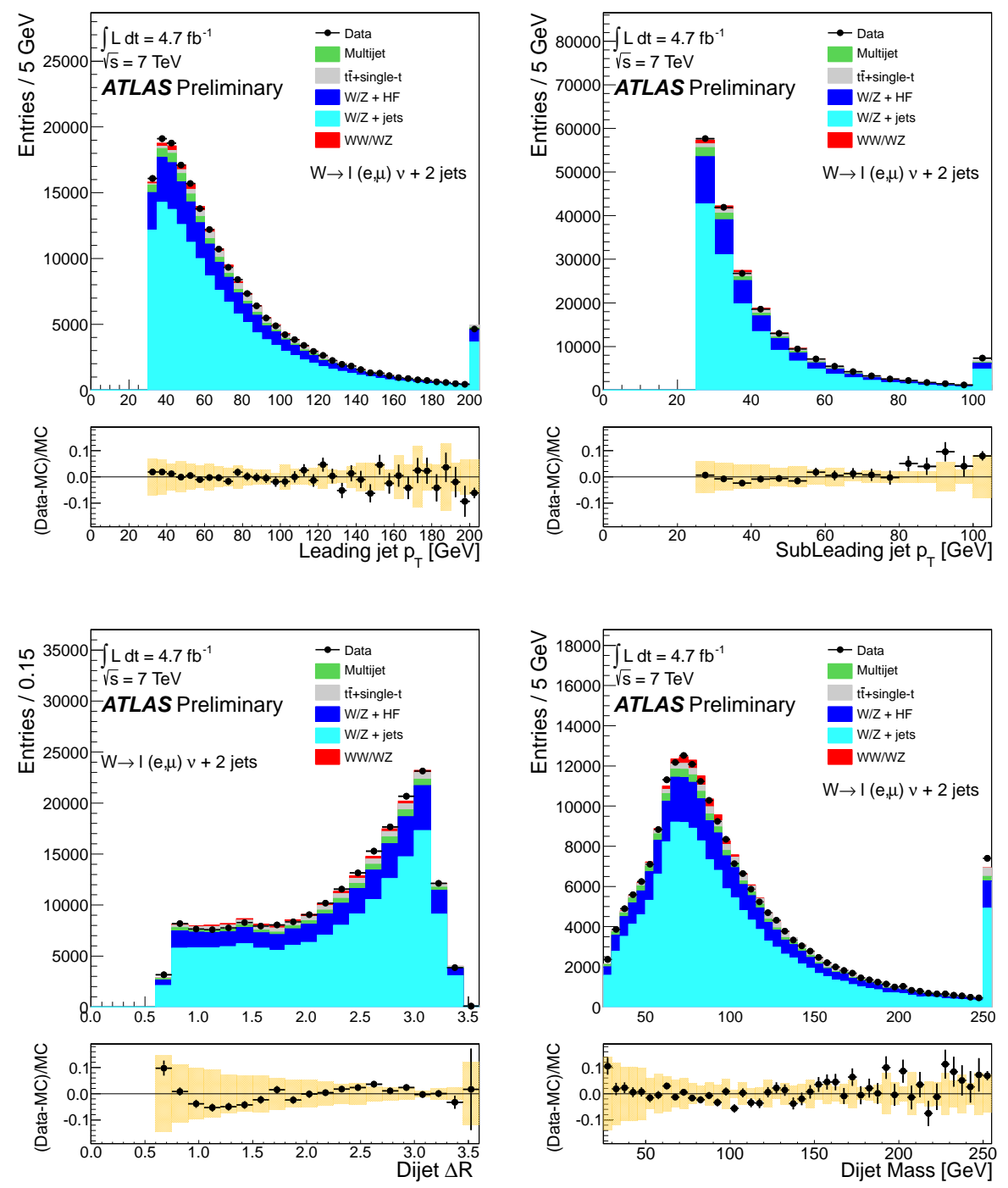

Figure 1: Comparison between data and MC expectation for four kinematic variables [8], after the event selection described in the text. The multi-jet background is estimated using a data-driven method. The yellow bands in the bottom portions of the plots give the systematic uncertainty only due to the jet energy scale.

experiments, most importantly the jet energy scale (JES), the shape of the multi-jet background, and the effect of limited MC statistics.

The results of the fit are shown in Fig. 3. A cross-section of $\sigma(p p \rightarrow W W / W Z)=72 \pm 9$ (stat.) \pm 15 (syst.) \pm 13 (MC statistics) pb is measured. This is consistent with the NLO SM expectation of $63.4 \pm 2.6 \mathrm{pb}$. Besides the $18 \%$ systematic from limited MC statistics, the other most significant systematics are from the JES effect on the dijet-mass spectrum (12\%) and the normalization of V+jets (11\%). The observed (statistical plus systematic) significance of the measurement is estimated using pseudo-experiments, and found to be $3.3 \sigma$, with a $3.0 \sigma$ expected significance. 

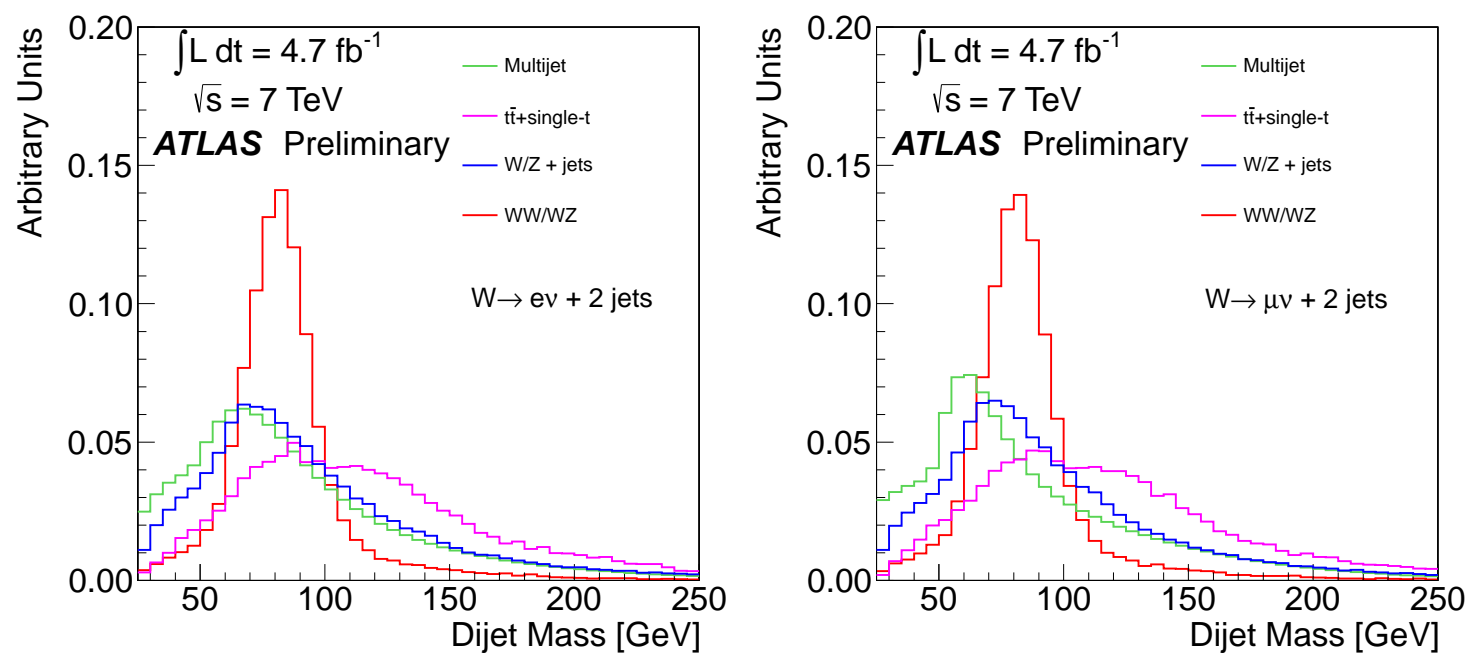

Figure 2: The templates for the signal and the different background components that are used in the fit to the dijet mass distribution [8]. The electron (muon) channel are shown on the left (right).

\section{Summary}

ATLAS measures the cross-section $\sigma(p p \rightarrow W W / W Z)=72 \pm 9$ (stat.) \pm 15 (syst.) \pm 13 (MC stat.) pb in the semi-leptonic $\ell v j j$ channel at $\sqrt{s}=7 \mathrm{TeV}$, in good agreement with the $\mathrm{SM}$ expectation. This measurement has a significance of $3.3 \sigma$, and represents the first evidence by ATLAS for diboson production in a semi-leptonic channel.

\section{References}

[1] ATLAS Collaboration, Measurement of ZZ production in pp collisions at $\sqrt{s}=7 \mathrm{TeV}$ and limits on anomalous ZZZ and ZZ $\gamma$ couplings with the ATLAS detector, JHEP 03 (2013) 128 [arXiv:1211.6096 [hep-ex]].

[2] ATLAS Collaboration, Measurement of $W^{ \pm} Z$ production in proton-proton collisions at $\sqrt{s}=7 \mathrm{TeV}$ with the ATLAS detector, Eur. Phys. J. C 72 (2012) 2173 [arXiv: 1208.1390 [hep-ex] ].

[3] ATLAS Collaboration, Measurement of $W^{+} W^{-}$production in pp collisions at $\sqrt{s}=7 \mathrm{TeV}$ with the ATLAS detector and limits on anomalous WWZ and WW $\gamma$ couplings, Phys. Rev. D 87 (2013) 112001 [arXiv:1210.2979 [hep-ex]].

[4] CMS Collaboration, Measurement of the WW, WZ and ZZ cross sections at CMS, CMS-PAS-EWK-11-010.

[5] CMS Collaboration, Measurement of the ZZ production cross section and search for anomalous couplings in $2 \ell 2 \ell^{\prime}$ final states in pp collisions at $\sqrt{s}=7$ TeV, JHEP 01 (2013) 063 [arXiv:1211.4890 [hep-ex]].

[6] CMS Collaboration, Measurement of the $W^{+} W^{-}$cross section in pp collisions at $\sqrt{s}=7$ TeV and limits on anomalous $W W \gamma$ and $W W Z$ couplings, CERN-PH-EP-2013-075, arXiv:1306.1126 [hep-ex]. 

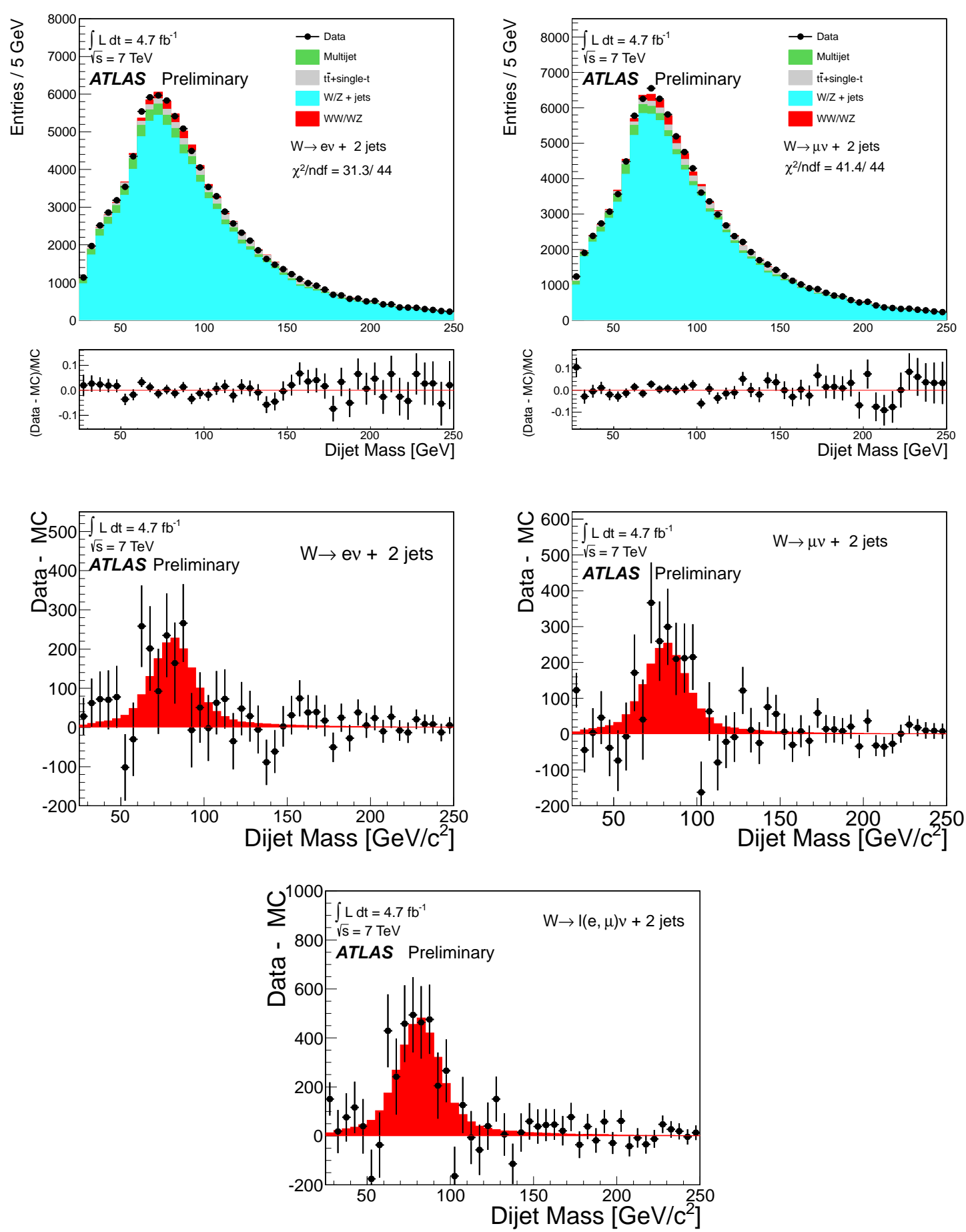

Figure 3: The results of the fit to the dijet mass distribution [8]. The top two plots show the fitted signal plus background compared to the data, for the electron and muon channels. The bottom three plots show the background-subtracted data compared to the fitted signal, for the electron and muon channels separately and for the combination of the channels. 
[7] CMS Collaboration, Measurement of the $W^{+} W^{-}$and ZZ production cross sections in pp collisions at $\sqrt{s}=8 \mathrm{TeV}$, Phys. Lett. B 721 (2013) 190 [arXiv:1301.4698 [hep-ex]].

[8] ATLAS Collaboration, Measurement of the combined WW and WZ production cross section in the semileptonic final state in proton-proton collisions at $\sqrt{s}=7$ TeV with the ATLAS detector, ATLAS-CONF-2012-157, http://cds.cern.ch/record/1493586.

[9] CMS Collaboration, Measurement of the sum of $W W$ and $W Z$ production with $W+$ dijet events in $p p$ collisions at $\sqrt{s}=7$ TeV, Eur. Phys. J. C 73 (2013) 2283 [arXiv: 1210.7544 [hep-ex] ].

[10] J.M. Campbell, R.K. Ellis, C. Williams, Vector boson pair production at the LHC, JHEP 07 (2011) 18 [arXiv:1105.0020 [hep-ph]].

[11] S. Agostinelli et al, GEANT4 - a simulation toolkit, NIM A506 (2003) 250.

[12] ATLAS Collaboration, The ATLAS Experiment at the CERN Large Hadron Collider, JINST 3 (2008) S08003.

[13] M. Cacciari, G.P. Salam, G. Soyez, The anti- $k_{t}$ jet clustering algorithm, JHEP 04 (2008) 63 [arXiv:0802.1189 [hep-ph]]. 